\title{
Stability of Analytical and Numerical Solutions for Nonlinear Stochastic Delay Differential Equations with Jumps
}

\author{
Qiyong $\mathrm{Li}^{1,2}$ and Siqing Gan ${ }^{1}$ \\ ${ }^{1}$ School of Mathematical Sciences and Computing Technology, Central South University, Changsha, \\ Hunan 410075, China \\ ${ }^{2}$ Department of Mathematics, Huaihua University, Huaihua, Hunan 418008, China
}

Correspondence should be addressed to Qiyong Li, lihaihust@yahoo.com.cn

Received 19 November 2011; Accepted 30 November 2011

Academic Editor: Shaher Momani

Copyright (C) 2012 Q. Li and S. Gan. This is an open access article distributed under the Creative Commons Attribution License, which permits unrestricted use, distribution, and reproduction in any medium, provided the original work is properly cited.

This paper is concerned with the stability of analytical and numerical solutions for nonlinear stochastic delay differential equations (SDDEs) with jumps. A sufficient condition for mean-square exponential stability of the exact solution is derived. Then, mean-square stability of the numerical solution is investigated. It is shown that the compensated stochastic $\theta$ methods inherit stability property of the exact solution. More precisely, the methods are mean-square stable for any stepsize $\Delta t=\tau / m$ when $1 / 2 \leq \theta \leq 1$, and they are exponentially mean-square stable if the stepsize $\Delta t \in\left(0, \Delta t_{0}\right)$ when $0 \leq \theta<1$. Finally, some numerical experiments are given to illustrate the theoretical results.

\section{Introduction}

Models that incorporate jumps have become increasingly popular in finance and several areas of science and engineering. In particular, they are used in mathematical finance in order to simulate asset prices, interest rates, and volatilities [1,2]. Jump models also arise in many other application areas and have proved successful at describing unexpected, abrupt changes of state [3]. So, it is valuable to investigate the properties of the solutions of these problems.

As is well known, explicit solutions of stochastic differential equations can rarely be obtained. It is necessary to construct efficient numerical methods to solve these equations. In recent years, many researchers worked on the construction of numerical schemes for stochastic ordinary differential equations (SODEs) (see $[4,5]$, and their references) and stochastic delay differential equations (SDDEs), see, for example, [6-11] and references therein. For SODEs with jumps, the strong convergence and mean-square stability of some 
semi-implicit numerical methods are investigated in [12-15]. A compensated split-step backward Euler method for SODEs with jumps is introduced in [12] and proved to satisfy a better stability property than the split-step backward Euler method.

For SDDEs with jumps, most of the existing work is concerned about convergence property of numerical methods, see, for example, [16-19]. There are few results on stability property, which motivates our work. In [20], Tan and Wang investigated the mean-square stability of the explicit Euler method for linear SDDEs with jumps. The aim of our paper is to investigate the mean-square stability of the compensated stochastic $\theta$ methods for nonlinear SDDEs with jumps.

This paper is organized as follows. In Section 2, we obtain a stability result for the analytical solution of (2.1). In Section 3, the compensated stochastic $\theta$ methods are constructed to solve problem (2.1). In Section 4, our main results will be stated and proved. It is shown that the compensated stochastic $\theta$ methods inherit mean-square stability of the exact solution. More precisely, the methods are mean-square stable for any stepsize $\Delta t=\tau / \mathrm{m}$ when $1 / 2 \leq \theta \leq 1$, and they are exponentially mean-square stable if the stepsize $\Delta t \in\left(0, \Delta t_{0}\right)$ when $0 \leq \theta<1$. Moreover, when $\theta=1$, the method is exponentially mean-square stable for every stepsize $\Delta t=\tau / \mathrm{m}$. Finally, some numerical experiments are reported to illustrate the theoretical results.

\section{Stability of the Analytical Solution}

Throughout this paper, we let $\mathcal{C}\left([-\tau, 0] ; R^{d}\right)$ denote the family of continuous functions from $[-\tau, 0]$ to $R^{d}$ equipped with the norm $\|\varphi\|:=\sup _{-\tau \leq s \leq 0}|\varphi(s)|$, where $|\cdot|$ is the Euclidean norm in $\mathbb{R}^{d}$. Denoted by $\mathcal{C}_{\Psi_{0}}^{b}\left([-\tau, 0] ; \mathbb{R}^{d}\right)$ the family of all bounded, $\mathcal{F}_{0}$ measurable, $\mathcal{C}\left([-\tau, 0] ; R^{d}\right)$ valued stochastic variables. The inner product of $x, y$ in $\mathbb{R}^{d}$ is denoted by $\langle x, y\rangle$. If $A$ is a vector or matrix, its transpose is denoted by $A^{T}$. If $A$ is a matrix, its trace norm is denoted by $|A|=\sqrt{\operatorname{trace}\left(A^{T} A\right)}$.

We consider the nonlinear SDDEs with jumps in Itô's sense of the form:

$$
\begin{aligned}
d x(t)= & f(x(t), x(t-\tau)) d t+g(x(t), x(t-\tau)) d W(t) \\
& +h\left(x\left(t^{-}\right), x\left(t^{-}-\tau\right)\right) d N(t), \quad t>0,
\end{aligned}
$$

with initial data $x(t)=\varphi(t), t \in[-\tau, 0]$ and $\tau>0$ is a constant, $x\left(t^{-}\right)$denotes $\lim _{s \rightarrow t^{-}} x(s)$, $f: \mathbb{R}^{d} \times \mathbb{R}^{d} \rightarrow \mathbb{R}^{d}, g: \mathbb{R}^{d} \times \mathbb{R}^{d} \rightarrow \mathbb{R}^{d \times m}, h: \mathbb{R}^{d} \times \mathbb{R}^{d} \rightarrow \mathbb{R}^{d}$ are continuous functions, and $\varphi(t) \in \mathcal{C}_{\mathcal{F}_{0}}^{b}\left([-\tau, 0] ; \mathbb{R}^{d}\right) . W(t)$ is an $m$-dimensional Wiener process defined on the complete probability space $\left(\Omega, \mathcal{F},\left\{\mathcal{F}_{t}\right\}_{t \geq 0}, P\right)$ with a filtration satisfying the usual conditions (i.e., it is increasing and right continuous while $\mathcal{F}_{0}$ contains all $P$-null sets). $N(t)$ is a scalar Poisson process with parameter $\lambda$ defined on the same probability space. Assume that $W(t)$ and $N(t)$ are independent of $\mathcal{F}_{0}$. Moreover, we assume that $\varphi(t)$ is $\mathcal{F}_{0}$-measurable and right continuous with $\mathbb{E}\|\varphi\|^{2}<\infty$. We also assume that $f(0,0)=0, g(0,0)=0$ and $h(0,0)=0$, so problem $(2.1)$ admits a zero solution $x(t) \equiv 0$.

Definition 2.1 (see [21]). The zero solution of (2.1) is said to be pth moment exponentially stable if there is a pair of positive constants $\lambda$ and $C$ such that

$$
\mathbb{E}|x(t)|^{p} \leq C\|\varphi\| \exp ^{-\lambda\left(t-t_{0}\right)}, \quad t \geq t_{0},
$$


for all $\varphi(t) \in \mathcal{C}_{q_{0}}^{b}\left([-\tau, 0] ; \mathbb{R}^{d}\right)$. When $p=2$, it is usually said to be exponentially mean-square stable.

Now, we establish a mean-square stability condition for problem (2.1).

Theorem 2.2. Suppose that there are some constants $\alpha_{i}, \beta_{i}, \gamma_{i}, i=1,2$, such that

$$
\begin{gathered}
\left\langle x_{1}-x_{2}, f\left(x_{1}, y\right)-f\left(x_{2}, y\right)\right\rangle \leq \alpha_{1}\left|x_{1}-x_{2}\right|^{2}, \\
\left|f\left(x, y_{1}\right)-f\left(x, y_{2}\right)\right| \leq \alpha_{2}\left|y_{1}-y_{2}\right|, \\
\left|g\left(x_{1}, y_{1}\right)-g\left(x_{2}, y_{2}\right)\right|^{2} \leq \beta_{1}\left|x_{1}-x_{2}\right|^{2}+\beta_{2}\left|y_{1}-y_{2}\right|^{2}, \\
\left|h\left(x_{1}, y_{1}\right)-h\left(x_{2}, y_{2}\right)\right|^{2} \leq \gamma_{1}\left|x_{1}-x_{2}\right|^{2}+\gamma_{2}\left|y_{1}-y_{2}\right|^{2},
\end{gathered}
$$

for all $x_{1}, y_{1}, x_{2}, y_{2} \in \mathbb{R}^{d}$. If

$$
\alpha=2 \alpha_{1}+2 \alpha_{2}+\beta_{1}+\beta_{2}+\lambda\left(1+2 \gamma_{1}+2 \gamma_{2}\right)<0,
$$

then the zero solution of (2.1) is exponentially mean-square stable.

Proof. Let $t \geq 0, \delta>0$, it follows from Itô's formula that

$$
\begin{aligned}
|x(t+\delta)|^{2}=|x(t)|^{2} & +\int_{t}^{t+\delta}\left(2\langle x(s), f(x(s), x(s-\tau))\rangle+|g(x(s), x(s-\tau))|^{2}\right) d s \\
& +\int_{t}^{t+\delta} 2\langle x(s), g(x(s), x(s-\tau))\rangle d W(s) \\
& +\int_{t}^{t+\delta}\left(2\left\langle x\left(s^{-}\right), h\left(x\left(s^{-}\right), x\left(s^{-}-\tau\right)\right)\right\rangle+\left|h\left(x\left(s^{-}\right), x\left(s^{-}-\tau\right)\right)\right|^{2}\right) d \widetilde{N}(s) \\
& +\lambda \int_{t}^{t+\delta}\left(2\langle x(s), h(x(s), x(s-\tau))\rangle+|h(x(s), x(s-\tau))|^{2}\right) d s,
\end{aligned}
$$

where $\widetilde{N}(t)=N(t)-\lambda t$. Taking expectation and using the properties of Itô integral give

$$
\begin{aligned}
\mathbb{E}|x(t+\delta)|^{2}= & \mathbb{E}|x(t)|^{2}+\mathbb{E} \int_{t}^{t+\delta}\left(2\langle x(s), f(x(s), x(s-\tau))\rangle+|g(x(s), x(s-\tau))|^{2}\right) d s \\
& +\lambda \mathbb{E} \int_{t}^{t+\delta}\left(2\langle x(s), h(x(s), x(s-\tau))\rangle+|h(x(s), x(s-\tau))|^{2}\right) d s .
\end{aligned}
$$


From (2.3) and (2.4), we have

$$
\begin{aligned}
2 \mathbb{E}\langle x(s), f(x(s), x(s-\tau))\rangle= & 2 \mathbb{E}\langle x(s), f(x(s), x(s-\tau))-f(0, x(s-\tau))\rangle \\
& +2 \mathbb{E}\langle x(s), f(0, x(s-\tau))\rangle \\
\leq & 2 \alpha_{1} \mathbb{E}|x(s)|^{2}+2 \alpha_{2} \mathbb{E}|x(s) \| x(s-\tau)| \\
\leq & 2 \alpha_{1} \mathbb{E}|x(s)|^{2}+2 \alpha_{2} \sup _{s-\tau \leq u \leq s} \mathbb{E}|x(u)|^{2} .
\end{aligned}
$$

Similarly, (2.5) and (2.6) yield

$$
\begin{gathered}
\mathbb{E}|g(x(s), x(s-\tau))|^{2} \leq\left(\beta_{1}+\beta_{2}\right) \sup _{s-\tau \leq u \leq s} \mathbb{E}|x(u)|^{2}, \\
\mathbb{E}|h(x(s), x(s-\tau))|^{2} \leq\left(\gamma_{1}+\gamma_{2}\right) \sup _{s-\tau \leq u \leq s} \mathbb{E}|x(u)|^{2}, \\
2 \mathbb{E}\langle x(s), h(x(s), x(s-\tau))\rangle \leq\left(1+\gamma_{1}+\gamma_{2}\right) \sup _{s-\tau \leq u \leq s} \mathbb{E}|x(u)|^{2} .
\end{gathered}
$$

Substituting (2.10)-(2.11) into (2.9) yields

$$
\begin{aligned}
& \mathbb{E}|x(t+\delta)|^{2} \\
& \quad \leq \mathbb{E}|x(t)|^{2}+\int_{t}^{t+\delta}\left(2 \alpha_{1} \mathbb{E}|x(s)|^{2}+\left(2 \alpha_{2}+\beta_{1}+\beta_{2}+\lambda\left(1+2 \gamma_{1}+2 \gamma_{2}\right)\right) \sup _{s-\tau \leq u \leq s} \mathbb{E}|x(u)|^{2}\right) d s .
\end{aligned}
$$

Let $v(t)=\mathbb{E}|x(t)|^{2}, \beta=2 \alpha_{2}+\beta_{1}+\beta_{2}+\lambda\left(1+2 \gamma_{1}+2 \gamma_{2}\right)$, we have

$$
D^{+} v(t) \leq 2 \alpha_{1} v(t)+\beta \sup _{t-\tau \leq u \leq t} v(u)
$$

where

$$
D^{+} v(t)=\limsup _{\delta \searrow 0} \frac{v(t+\delta)-v(t)}{\delta}
$$

Moreover, $\alpha<0$ implies $-2 \alpha_{1}>\beta \geq 0$. By Lemma 1.1 in [22], there exist positive constants $v$ and $k$ such that

$$
v(t) \leq k e^{-v t}, \quad t \geq 0
$$

Hence, the theorem is proven.

Based on the above result, we are going to study the stability of numerical methods for (2.1) in the following sections. 


\section{Compensated Stochastic $\theta$ Methods for Nonlinear SDDEs with Jumps}

Since the compensated Poisson process $\widetilde{N}(t)=N(t)-\lambda t$ is a martingale satisfying the property

$$
\mathbb{E}(\widetilde{N}(t+s)-\widetilde{N}(t))=0, \quad \mathbb{E}|\widetilde{N}(t+s)-\widetilde{N}(t)|^{2}=\lambda s, \quad t, s \geq 0,
$$

we rewrite problem (2.1) in an equivalent form:

$$
\begin{gathered}
d x(t)=\tilde{f}(x(t), x(t-\tau)) d t+g(x(t), x(t-\tau)) d W(t) \\
+h\left(x\left(t^{-}\right), x\left(t^{-}-\tau\right)\right) d \widetilde{N}(t), \quad t>0,
\end{gathered}
$$

where $\tilde{f}(x, y)$ is defined as

$$
\tilde{f}(x, y):=f(x, y)+\lambda h(x, y)
$$

Applying the stochastic $\theta$ methods to (3.2) leads to the following compensated stochastic $\theta$ methods:

$$
\begin{aligned}
X_{n+1}= & X_{n}+(1-\theta) \tilde{f}\left(X_{n}, X_{n-m}\right) \Delta t+\theta \tilde{f}\left(X_{n+1}, X_{n-m+1}\right) \Delta t \\
& +g\left(X_{n}, X_{n-m}\right) \Delta W_{n}+h\left(X_{n}, X_{n-m}\right) \Delta \widetilde{N}_{n} .
\end{aligned}
$$

Here, $X_{n}$ denotes that the approximation to $x\left(t_{n}\right), \theta$ is a parameter with $0 \leq \theta \leq 1, \Delta t:=t_{n+1}-t_{n}$ is the stepsize which satisfies $\tau=m \Delta t$ for a positive integer $m, \Delta W_{n}:=W\left(t_{n+1}\right)-W\left(t_{n}\right)$ and $\Delta \widetilde{N}_{n}:=\widetilde{N}\left(t_{n+1}\right)-\widetilde{N}\left(t_{n}\right)$. In particular,

$$
X_{l}=\varphi(l \Delta t), \quad l \leq 0
$$

Note that for $\theta>0$, the numerical solutions in (3.4) are defined by implicit equations. However, due to the one-sided Lipschitz condition (2.3), (3.4) has a unique solution, with probability one, for all $\theta \Delta t\left(\alpha_{1}+\lambda \sqrt{\gamma_{1}}\right)<1$, see, for example, [23, Theorem 14.2] and (19) in [12].

Remark 3.1. Since $\alpha<0$ implies $\alpha_{1}+\lambda \sqrt{\gamma_{1}}<0$, then the compensated stochastic $\theta$ methods (3.4) produce a well-defined, unique solution if the stability condition $\alpha<0$ holds.

Definition 3.2. For a give stepsize $\Delta t=\tau / m$, a numerical method on the nonlinear SDDEs with jumps (2.1) is said to be exponentially mean-square stable, if there exist positive constants $N$ and $\gamma$, such that the numerical solution $X_{n}$ produced by this method satisfies

$$
\mathbb{E}\left|X_{n}\right|^{2} \leq N\|\varphi\| e^{-\gamma\left(t_{n}-t_{0}\right)},
$$

for all initial data $\varphi \in \mathcal{C}_{\Psi_{0}}^{b}\left([-\tau, 0] ; \mathbb{R}^{d}\right)$. 
Definition 3.3. For a give stepsize $\Delta t=\tau / m$, a numerical method on the nonlinear SDDEs with jumps (2.1) is said to be mean-square stable if the numerical solution $X_{n}$ produced by this method satisfies

$$
\lim _{n \rightarrow \infty} \mathbb{E}\left|X_{n}\right|^{2}=0
$$

\section{Stability Analysis of the Numerical Solutions}

In this section, we study mean-square stability and exponentially mean-square stability of the compensated stochastic $\theta$ methods (3.4). Now, we present the main results of the paper.

Theorem 4.1. Suppose that (2.3)-(2.7) hold. If $1 / 2 \leq \theta \leq 1$, then the compensated stochastic $\theta$ methods are mean-square stable for every stepsize $\Delta t=\tau / \mathrm{m}$.

Proof. It follows from (3.4) that

$$
\begin{aligned}
\left|X_{n+1}-\theta \Delta t \tilde{f}\left(X_{n+1}, X_{n-m+1}\right)\right|^{2}= & \left|X_{n}-\theta \Delta t \tilde{f}\left(X_{n}, X_{n-m}\right)\right|^{2}+2 \Delta t\left\langle X_{n}, \tilde{f}\left(X_{n}, X_{n-m}\right)\right\rangle \\
& +(\Delta t)^{2}(1-2 \theta)\left|\tilde{f}\left(X_{n}, X_{n-m}\right)\right|^{2}+\left|g\left(X_{n}, X_{n-m}\right) \Delta W_{n}\right|^{2} \\
& +\left|h\left(X_{n}, X_{n-m}\right) \Delta \widetilde{N}_{n}\right|^{2}+M_{n},
\end{aligned}
$$

where

$$
\begin{aligned}
M_{n}= & 2\left\langle X_{n}+(1-\theta) \Delta t \tilde{f}\left(X_{n}, X_{n-m}\right), g\left(X_{n}, X_{n-m}\right) \Delta W_{n}\right\rangle \\
& +2\left\langle X_{n}+(1-\theta) \Delta t \tilde{f}\left(X_{n}, X_{n-m}\right), h\left(X_{n}, X_{n-m}\right) \Delta \widetilde{N}_{n}\right\rangle \\
& +2\left\langle g\left(X_{n}, X_{n-m}\right) \Delta W_{n}, h\left(X_{n}, X_{n-m}\right) \Delta \widetilde{N}_{n}\right\rangle .
\end{aligned}
$$

Thus, for $1 / 2 \leq \theta \leq 1$, we have

$$
\begin{aligned}
\left|X_{n+1}-\theta \Delta t \tilde{f}\left(X_{n+1}, X_{n-m+1}\right)\right|^{2} \leq & \left|X_{n}-\theta \Delta t \tilde{f}\left(X_{n}, X_{n-m}\right)\right|^{2}+2 \Delta t\left\langle X_{n}, \tilde{f}\left(X_{n}, X_{n-m}\right)\right\rangle \\
& +\left|g\left(X_{n}, X_{n-m}\right) \Delta W_{n}\right|^{2}+\left|h\left(X_{n}, X_{n-m}\right) \Delta \widetilde{N}_{n}\right|^{2}+M_{n} .
\end{aligned}
$$

It follows from (2.3), (2.4), and (2.6) that

$$
\begin{aligned}
2\left\langle X_{n}, \tilde{f}\left(X_{n}, X_{n-m}\right)\right\rangle & =2\left\langle X_{n}, f\left(X_{n}, X_{n-m}\right)\right\rangle+2 \lambda\left\langle X_{n}, h\left(X_{n}, X_{n-m}\right)\right\rangle \\
& \leq 2 \alpha_{1}\left|X_{n}\right|^{2}+\alpha_{2}\left|X_{n}\right|^{2}+\alpha_{2}\left|X_{n-m}\right|^{2}+\lambda\left(\left|X_{n}\right|^{2}+\gamma_{1}\left|X_{n}\right|^{2}+\gamma_{2}\left|X_{n-m}\right|^{2}\right) .
\end{aligned}
$$


Note that $\mathbb{E}\left(\Delta W_{n}\right)=0, \mathbb{E}\left(\Delta \widetilde{N}_{n}\right)=0$ and $\mathbb{E}\left(\Delta \widetilde{N}_{n}\right)^{2}=\lambda \Delta t$. Furthermore, $X_{n}$ and $X_{n-m}$ are all $\mathcal{F}_{t_{n}}$-measurable. Therefore, we can easily obtain

$$
\begin{aligned}
\mathbb{E}\left|g\left(X_{n}, X_{n-m}\right) \Delta W_{n}\right|^{2} & =\Delta t \mathbb{E}\left|g\left(X_{n}, X_{n-m}\right)\right|^{2}, \\
\mathbb{E}\left|h\left(X_{n}, X_{n-m}\right) \Delta \widetilde{N}_{n}\right|^{2} & =\lambda \Delta t \mathbb{E}\left|h\left(X_{n}, X_{n-m}\right)\right|^{2}, \\
\mathbb{E} M_{n} & =0 .
\end{aligned}
$$

Taking expectation on both sides of (4.3) and substituting (4.4)-(4.7) into (4.3), we have

$$
\begin{aligned}
\mathbb{E} \mid X_{n+1}- & \left.\theta \Delta t \tilde{f}\left(X_{n+1}, X_{n-m+1}\right)\right|^{2} \\
\leq & \mathbb{E}\left|X_{n}-\theta \Delta t \tilde{f}\left(X_{n}, X_{n-m}\right)\right|^{2}+\Delta t\left(2 \alpha_{1}+\alpha_{2}+\beta_{1}+\lambda+2 \lambda \gamma_{1}\right) \mathbb{E}\left|X_{n}\right|^{2} \\
& +\Delta t\left(\alpha_{2}+\beta_{2}+2 \lambda \gamma_{2}\right) \mathbb{E}\left|X_{n-m}\right|^{2}
\end{aligned}
$$

Consequently, by the recursion of inequality (4.8), we have

$$
\begin{aligned}
\mathbb{E} \mid X_{n+1}- & \left.\theta \Delta t \tilde{f}\left(X_{n+1}, X_{n-m+1}\right)\right|^{2} \\
\leq & \mathbb{E}\left|X_{n}-\theta \Delta t \tilde{f}\left(X_{n}, X_{n-m}\right)\right|^{2} \\
& \quad+\Delta t\left(2 \alpha_{1}+\alpha_{2}+\beta_{1}+\lambda+2 \lambda \gamma_{1}\right) \mathbb{E}\left|X_{n}\right|^{2}+\Delta t\left(\alpha_{2}+\beta_{2}+2 \lambda \gamma_{2}\right) \mathbb{E}\left|X_{n-m}\right|^{2} \\
\leq & \mathbb{E}\left|X_{n-1}-\theta \Delta t \tilde{f}\left(X_{n-1}, X_{n-m-1}\right)\right|^{2} \\
& +\Delta t\left(2 \alpha_{1}+\alpha_{2}+\beta_{1}+\lambda+2 \lambda \gamma_{1}\right) \sum_{j=n-1}^{n} \mathbb{E}\left|X_{j}\right|^{2}+\Delta t\left(\alpha_{2}+\beta_{2}+2 \lambda \gamma_{2}\right) \sum_{j=n-1}^{n} \mathbb{E}\left|X_{j-m}\right|^{2} \\
\leq & \ldots \\
\leq & \mathbb{E}\left|X_{0}-\theta \Delta t \tilde{f}\left(X_{0}, X_{-m}\right)\right|^{2} \\
& +\Delta t\left(2 \alpha_{1}+\alpha_{2}+\beta_{1}+\lambda+2 \lambda \gamma_{1}\right) \sum_{j=0}^{n} \mathbb{E}\left|X_{j}\right|^{2}+\Delta t\left(\alpha_{2}+\beta_{2}+2 \lambda \gamma_{2}\right) \sum_{j=0}^{n} \mathbb{E}\left|X_{j-m}\right|^{2} .
\end{aligned}
$$

Noting that $\sum_{j=0}^{n} \mathbb{E}\left|X_{j-m}\right|^{2}=\sum_{j=-m}^{-1} \mathbb{E}\left|X_{j}\right|^{2}+\sum_{j=0}^{n-m} \mathbb{E}\left|X_{j}\right|^{2}$ and $\tau=m \Delta t$, we derive from (4.9) that

$$
\begin{aligned}
& \mathbb{E}\left|X_{n+1}-\theta \Delta t \tilde{f}\left(X_{n+1}, X_{n-m+1}\right)\right|^{2} \\
& \leq \mathbb{E}\left|X_{0}-\theta \Delta t \tilde{f}\left(X_{0}, X_{-m}\right)\right|^{2}
\end{aligned}
$$




$$
\begin{aligned}
& +\Delta t\left(2 \alpha_{1}+2 \alpha_{2}+\beta_{1}+\beta_{2}+\lambda\left(1+2 \gamma_{1}+2 \gamma_{2}\right)\right) \sum_{j=0}^{n} \mathbb{E}\left|X_{j}\right|^{2}+\tau\left(\alpha_{2}+\beta_{2}+2 \lambda \gamma_{2}\right) \\
& \times \max _{-m \leq j \leq 0} \mathbb{E}\left|X_{j}\right|^{2} .
\end{aligned}
$$

Rearranging (4.10) and using the notation $\alpha$ in (2.7), we obtain

$$
\begin{aligned}
& \mathbb{E}\left|X_{n+1}-\theta \Delta t \tilde{f}\left(X_{n+1}, X_{n-m+1}\right)\right|^{2}-\alpha \Delta t \sum_{j=0}^{n} \mathbb{E}\left|X_{j}\right|^{2} \\
& \quad \leq E\left|X_{0}-\theta \Delta t \tilde{f}\left(X_{0}, X_{-m}\right)\right|^{2}+\left(\alpha_{2}+\beta_{2}+2 \lambda \gamma_{2}\right) \tau \max _{-m \leq j \leq 0} \mathbb{E}\left|X_{j}\right|^{2} .
\end{aligned}
$$

Since $\mathbb{E}\|\varphi\|^{2}<\infty$ and $\alpha<0$, we then derive that the series $\sum_{j=0}^{\infty} \mathbb{E}\left|X_{j}\right|^{2}$ is convergent, which implies $\lim _{n \rightarrow \infty} \mathbb{E}\left|X_{n}\right|^{2}=0$. Consequently, for $1 / 2 \leq \theta \leq 1$, the compensated stochastic $\theta$ methods are mean-square stable for any stepsize $\Delta t=\tau / \mathrm{m}$.

In order to investigate the exponential stability of the numerical methods, we need the following lemma which is Theorem 1 in [24].

Lemma 4.2 (see [24]). Suppose, for some fixed integer $N \geq 0$, that $t_{n}=t_{0}+n \Delta t$ for some $\Delta t>0$ and $\left\{v_{n}\right\}_{-N}^{\infty}$ is a sequence of positive numbers that satisfies

$$
\frac{v_{n+1}-v_{n}}{\Delta t} \leq-\alpha_{\Delta t} v_{n}+\beta_{\Delta t} \max _{j \in 2} v_{n+j} \quad \text { for } n \in \mathcal{N}
$$

with $N=0$ if $\beta_{\Delta t}=0$, where $2:=\{-N, \ldots,-1,0\}$. If

$$
0 \leq \beta_{\Delta t}<\alpha_{\Delta t}, \quad 0<\alpha_{\Delta t} \Delta t<1,
$$

then $v_{n} \leq\left\{\max _{j \in 2} v_{j}\right\} \exp \left\{-v^{+}\left(t_{n}-t_{0}\right)\right\}$, where $v^{+}>0$ is a constant.

Now, we present the result as follows.

Theorem 4.3. Suppose that (2.3)-(2.7) hold and the drift coefficient $f$ satisfies the linear growth condition, that is, there is a constant $D$ such that

$$
|f(x, y)|^{2} \leq D\left(|x|^{2}+|y|^{2}\right)
$$

Define $\Delta t_{1}=-\alpha / 2(1-\theta)^{2}\left(2 D+\lambda^{2}\left(\gamma_{1}+\gamma_{2}\right)\right), \Delta t_{2}=-\left(2 \alpha_{1}+\alpha_{2}+\beta_{1}+\lambda+2 \lambda \gamma_{1}\right) / 2(1-\theta)^{2}\left(D+\lambda^{2} \gamma_{1}\right)$ and $\Delta t_{3}=\inf \{\Delta t>0: P(\theta, \Delta t)<0\}$, where $P(\theta, \Delta t)=2(1-\theta)^{2}\left(D+\lambda^{2} \gamma_{1}\right)(\Delta t)^{2}+\left((1-\theta)\left(2 \alpha_{1}+\alpha_{2}+\right.\right.$ $\left.\left.\lambda+\lambda \gamma_{1}\right)+\beta_{1}+\lambda \gamma_{1}\right) \Delta t+1$. If $0 \leq \theta<1$, and the stepsize $\Delta t \in\left(0, \Delta t_{0}\right)$ with $\Delta t_{0}=\min \left\{\Delta t_{1}, \Delta t_{2}, \Delta t_{3}\right\}$, then the compensated stochastic $\theta$ methods are exponentially mean-square stable. 
Proof. We derive from (3.4) that

$$
\begin{aligned}
\mid X_{n+1} & -\left.\theta \Delta t \tilde{f}\left(X_{n+1}, X_{n-m+1}\right)\right|^{2} \\
& =\left|X_{n}+(1-\theta) \Delta t \tilde{f}\left(X_{n}, X_{n-m}\right)+g\left(X_{n}, X_{n-m}\right) \Delta W_{n}+h\left(X_{n}, X_{n-m}\right) \Delta \widetilde{N}_{n}\right|^{2} .
\end{aligned}
$$

Hence, we have

$$
\begin{aligned}
\left|X_{n+1}\right|^{2} \leq & \left|X_{n}\right|^{2}+(1-\theta)^{2}(\Delta t)^{2}\left|\tilde{f}\left(X_{n}, X_{n-m}\right)\right|^{2}+\left|g\left(X_{n}, X_{n-m}\right) \Delta W_{n}\right|^{2}+\left|h\left(X_{n}, X_{n-m}\right) \Delta \widetilde{N}_{n}\right|^{2} \\
& +2 \theta \Delta t\left\langle X_{n+1}, \tilde{f}\left(X_{n+1}, X_{n-m+1}\right)\right\rangle+2(1-\theta) \Delta t\left\langle X_{n}, \tilde{f}\left(X_{n}, X_{n-m}\right)\right\rangle+M_{n}
\end{aligned}
$$

where $M_{n}$ is defined as (4.2). From (2.6), (3.3), and (4.14), we obtain

$$
\begin{aligned}
\left|\tilde{f}\left(X_{n}, X_{n-m}\right)\right|^{2} & =\left|f\left(X_{n}, X_{n-m}\right)+\lambda h\left(X_{n}, X_{n-m}\right)\right|^{2} \\
& \leq 2\left(D\left(\left|X_{n}\right|^{2}+\left|X_{n-m}\right|^{2}\right)+\lambda^{2}\left(\gamma_{1}\left|X_{n}\right|^{2}+\gamma_{2}\left|X_{n-m}\right|^{2}\right)\right) .
\end{aligned}
$$

Substituting (4.4)-(4.7) and (4.17) into (4.16), and taking expectation, we have

$$
\begin{aligned}
\mathbb{E}\left|X_{n+1}\right|^{2} \leq & \mathbb{E}\left|X_{n}\right|^{2}+2(1-\theta)^{2}(\Delta t)^{2}\left(\left(D+\lambda^{2} \gamma_{1}\right) \mathbb{E}\left|X_{n}\right|^{2}+\left(D+\lambda^{2} \gamma_{2}\right) \mathbb{E}\left|X_{n-m}\right|^{2}\right) \\
& +\Delta t\left(\beta_{1} \mathbb{E}\left|X_{n}\right|^{2}+\beta_{2} \mathbb{E}\left|X_{n-m}\right|^{2}\right)+\lambda \Delta t\left(\gamma_{1} \mathbb{E}\left|X_{n}\right|^{2}+\gamma_{2} \mathbb{E}\left|X_{n-m}\right|^{2}\right) \\
& +\theta \Delta t\left(\left(2 \alpha_{1}+\alpha_{2}+\lambda\left(1+\gamma_{1}\right)\right) \mathbb{E}\left|X_{n+1}\right|^{2}+\left(\alpha_{2}+\lambda \gamma_{2}\right) \mathbb{E}\left|X_{n-m+1}\right|^{2}\right) \\
& +(1-\theta) \Delta t\left(\left(2 \alpha_{1}+\alpha_{2}+\lambda\left(1+\gamma_{1}\right)\right) \mathbb{E}\left|X_{n}\right|^{2}+\left(\alpha_{2}+\lambda \gamma_{2}\right) \mathbb{E}\left|X_{n-m}\right|^{2}\right)
\end{aligned}
$$

which yields

$$
\begin{aligned}
(1-\theta \Delta & \left.t\left(2 \alpha_{1}+\alpha_{2}+\lambda\left(1+\gamma_{1}\right)\right)\right) \mathbb{E}\left|X_{n+1}\right|^{2} \\
\leq & \left(1-\theta \Delta t\left(2 \alpha_{1}+\alpha_{2}+\lambda\left(1+\gamma_{1}\right)\right)\right) \mathbb{E}\left|X_{n}\right|^{2}+\left(\left(2 \alpha_{1}+\alpha_{2}+\beta_{1}+\lambda+2 \lambda \gamma_{1}\right) \Delta t\right. \\
& \left.+2(1-\theta)^{2}\left(D+\lambda^{2} \gamma_{1}\right)(\Delta t)^{2}\right) \mathbb{E}\left|X_{n}\right|^{2} \\
& +\left(\left(\alpha_{2}+\beta_{2}+2 \lambda \gamma_{2}\right) \Delta t+2(1-\theta)^{2}\left(D+\lambda^{2} \gamma_{2}\right)(\Delta t)^{2}\right)_{n-m \leq i \leq n-m+1} \mathbb{E}\left|X_{i}\right|^{2} .
\end{aligned}
$$

Hence,

$$
\frac{\mathbb{E}\left|X_{n+1}\right|^{2}-\mathbb{E}\left|X_{n}\right|^{2}}{\Delta t} \leq-A \mathbb{E}\left|X_{n}\right|^{2}+B \max _{n-m \leq i \leq n-m+1} \mathbb{E}\left|X_{i}\right|^{2}
$$


where

$$
\begin{gathered}
A=-\frac{2 \alpha_{1}+\alpha_{2}+\beta_{1}+\lambda+2 \lambda \gamma_{1}+2(1-\theta)^{2}\left(D+\lambda^{2} \gamma_{1}\right) \Delta t}{1-\theta \Delta t\left(2 \alpha_{1}+\alpha_{2}+\lambda\left(1+\gamma_{1}\right)\right)}, \\
B=\frac{\alpha_{2}+\beta_{2}+2 \lambda \gamma_{2}+2(1-\theta)^{2}\left(D+\lambda^{2} \gamma_{2}\right) \Delta t}{1-\theta \Delta t\left(2 \alpha_{1}+\alpha_{2}+\lambda\left(1+\gamma_{1}\right)\right)} .
\end{gathered}
$$

By Lemma 4.2, we derive that the methods are exponentially mean-square stable if

$$
0 \leq B<A, \quad 0<A \Delta t<1 .
$$

That is,

$$
\begin{gathered}
\Delta t<\frac{-\alpha}{2(1-\theta)^{2}\left(2 D+\lambda^{2}\left(\gamma_{1}+\gamma_{2}\right)\right)}, \\
\Delta t<-\frac{2 \alpha_{1}+\alpha_{2}+\beta_{1}+\lambda+2 \lambda \gamma_{1}}{2(1-\theta)^{2}\left(D+\lambda^{2} \gamma_{1}\right)}, \\
P(\theta, \Delta t)>0,
\end{gathered}
$$

where $P(\theta, \Delta t)=2(1-\theta)^{2}\left(D+\lambda^{2} \gamma_{1}\right)(\Delta t)^{2}+\left((1-\theta)\left(2 \alpha_{1}+\alpha_{2}+\lambda+\lambda \gamma_{1}\right)+\beta_{1}+\lambda \gamma_{1}\right) \Delta t+1$. Since $P(\theta, 0)=1$, there must exist $\Delta t_{3}>0$ such that $P(\theta, \Delta t)>0$ when $\Delta t<\Delta t_{3}$. On the other hand, if $P(\theta, \Delta t) \geq 0$ is always true, we then define $\Delta t_{3}$ as $\infty$. Therefore, let $\Delta t_{1}=-\alpha / 2(1-\theta)^{2}(2 D+$ $\left.\lambda^{2}\left(\gamma_{1}+\gamma_{2}\right)\right), \Delta t_{2}=-\left(2 \alpha_{1}+\alpha_{2}+\beta_{1}+\lambda+2 \lambda \gamma_{1}\right) / 2(1-\theta)^{2}\left(D+\lambda^{2} \gamma_{1}\right), \Delta t_{3}=\inf \{\Delta t>0: P(\theta, \Delta t)<0\}$ and $\Delta t_{0}=\min \left\{\Delta t_{1}, \Delta t_{2}, \Delta t_{3}\right\}$, then (4.22) holds when $\Delta t \in\left(0, \Delta t_{0}\right)$, which completes the proof of Theorem 4.3.

By the proof of Theorem 4.3, we can easily obtain the following result.

Theorem 4.4. Suppose that (2.3)-(2.7) hold. If $\theta=1$, then the compensated stochastic $\theta$-method is exponentially mean-square stable for every stepsize $\Delta t=\tau / \mathrm{m}$.

\section{Numerical Examples}

The purpose of this section is to illustrate our theoretical results presented in the previous section by numerical experiments. We first consider the following nonlinear scalar SDDEs with jumps:

$$
\begin{gathered}
d x(t)=\left(-4 x(t)-x^{3}(t)+x(t-1)\right) d t+\sin (x(t-1)) d W(t)-x\left(t^{-}\right) d N(t), \quad t>0, \\
x(t)=1, \quad t \in[-1,0]
\end{gathered}
$$

where $N(t)$ is a scalar Poisson process with parameter $\lambda=1$. In this case, (2.3)-(2.6) are satisfied with $\alpha_{1}=-4, \alpha_{2}=1, \beta_{1}=0, \beta_{2}=1, \gamma_{1}=1, \gamma_{2}=0$, and $\tau=1$. So we have $\alpha=-2$ in (2.7), which guarantees mean-square stability of the zero solution of (5.1) by Theorem 2.2. 


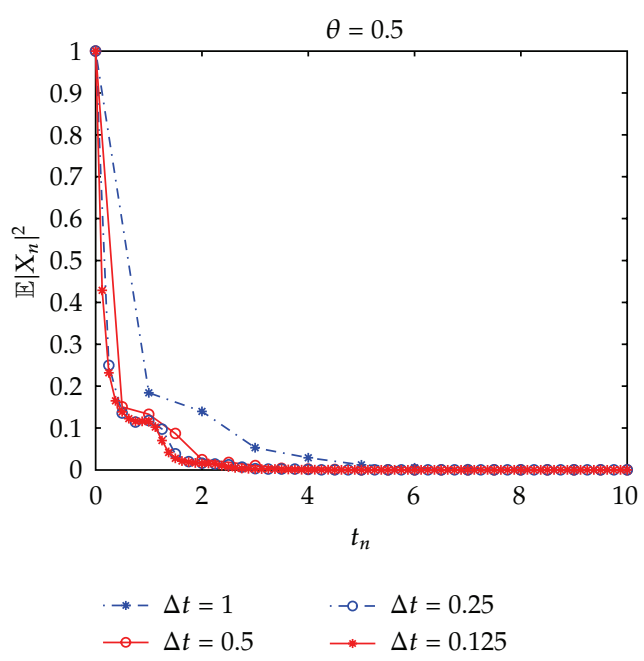

(a)

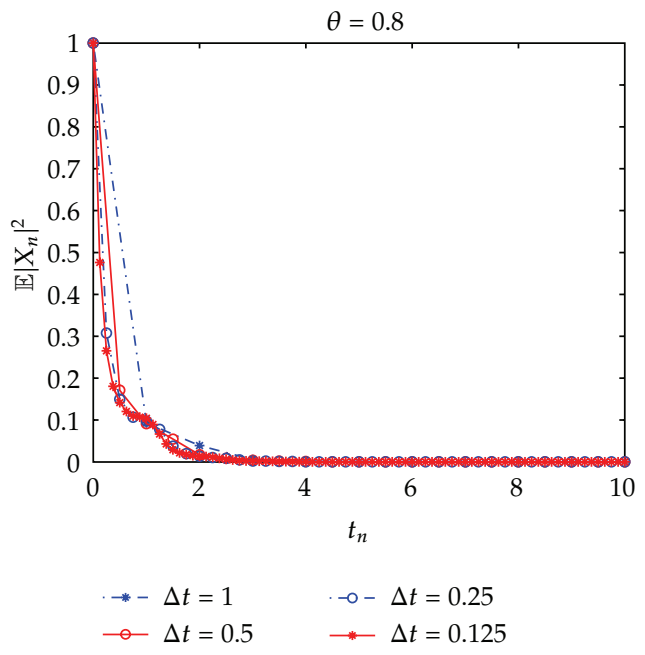

(b)

Figure 1: Fixed $\theta=0.5$ (a) and $\theta=0.8$ (b) with different stepsize $\Delta t=1 / 8,1 / 4,1 / 2,1$.

The following numerical experiments will show how the parameter $\theta$ and the stepsize $\Delta t$ influence the mean-square stability of the compensated stochastic $\theta$ methods. We simulate the expectation of $\left|X_{n}\right|^{2}$ by using 1000 trajectories, that is,

$$
\mathbb{E}\left|X_{n}\right|^{2} \approx \frac{1}{1000} \sum_{i=1}^{1000}\left|X_{n}\left(\omega_{i}\right)\right|^{2}
$$

Theorem 4.1 shows that the compensated stochastic $\theta$ methods are mean-square stable for every stepsize $\Delta t=\tau / m$ when $1 / 2 \leq \theta \leq 1$. In Figure 1 , we use (3.4) to solve (5.1) and choose the parameter $\theta$ with different values 0.5 and 0.8 , and we take the stepsize $\Delta t=$ $1 / 8,1 / 4,1 / 2$, and 1 , respectively. We can find that the compensated stochastic $\theta$ methods are mean-square stable with these stepsizes.

Theorem 4.3 shows that the compensated stochastic $\theta$ methods are exponentially mean-square stable if the stepsize $\Delta t \in\left(0, \Delta t_{0}\right)$ when $0 \leq \theta<1$. Now, we consider the following nonlinear scalar SDDEs with jumps:

$$
\begin{gathered}
d x(t)=-3 x(t) d t+\sin (x(t-1)) d W(t)-x\left(t^{-}-1\right) d N(t), \quad t>0, \\
x(t)=1, \quad t \in[-1,0],
\end{gathered}
$$

where $N(t)$ is a scalar Poisson process with parameter $\lambda=1$. (2.3)-(2.7) and (4.14) are satisfied with $\alpha_{1}=-3, \quad \alpha_{2}=0, \quad \beta_{1}=0, \quad \beta_{2}=1, \gamma_{1}=0, \quad \gamma_{2}=1, \quad \tau=1, \quad D=9$, and $\alpha=-2$. Therefore, the zero solution of (5.3) is exponentially mean-square stable. By Theorem 4.3, we calculate $\Delta t_{1}=1 / 19(1-\theta)^{2}, \Delta t_{2}=5 / 18(1-\theta)^{2}$, and $P(\theta, \Delta t)=18(1-\theta)^{2}(\Delta t)^{2}-5(1-\theta) \Delta t+1$. It is easy to see that $P(\theta, \Delta t)>0$ for every $\Delta t>0$, then we get $\Delta t_{3}=\infty$. Therefore, we obtain $\Delta t_{0}=\min \left\{\Delta t_{1}, \Delta t_{2}, \Delta t_{3}\right\}=1 / 19(1-\theta)^{2}$, which implies that the methods applied to (5.3) have less restrictions on the stepsize as the value of $\theta$ increases. Now, we use (3.4) to solve (5.3) and choose the parameter $\theta=0$ and $\theta=0.2$, and we take the stepsize $\Delta t=1 / 20,1 / 8,1 / 2$, and 


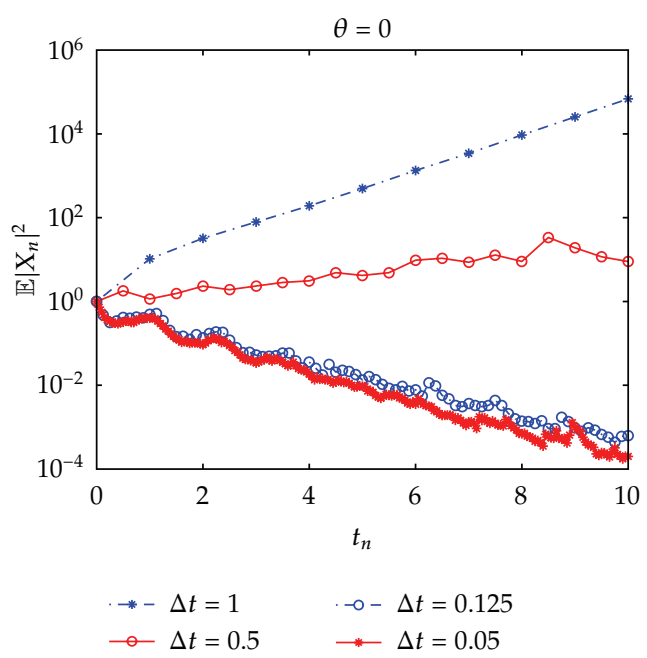

(a)

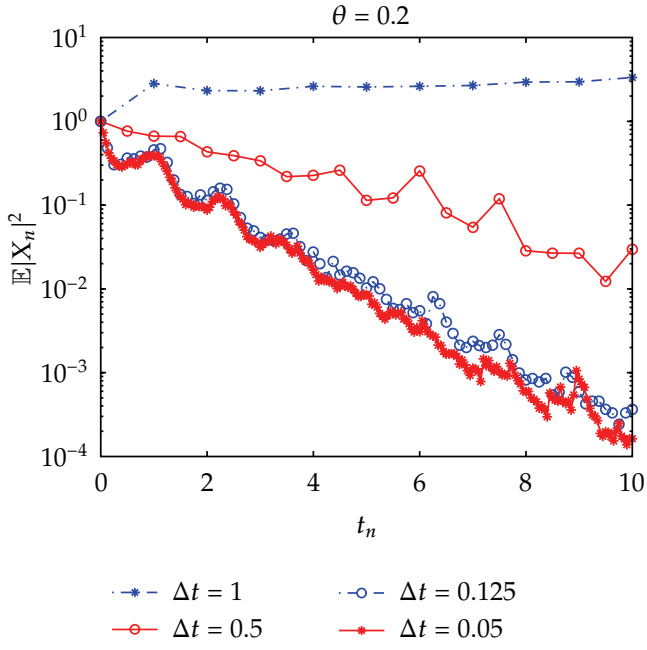

(b)

Figure 2: Fixed $\theta=0$ (a) and $\theta=0.2$ (b) with different stepsize $\Delta t=1 / 20,1 / 8,1 / 2,1$.

1, respectively. By Theorem 4.3, we compute that $\Delta t_{0}=0.0526$ when $\theta=0$ and $\Delta t_{0}=0.0822$ when $\theta=0.2$. Figure 2 indicates that both methods are exponentially mean-square stable if the stepsize $\Delta t=0.05$, which is well selected in $\Delta t \in\left(0, \Delta t_{0}\right)$. It also shows that the methods maybe stable when the stepsize is bigger than $\Delta t_{0}$, since both methods are stable when stepsize $\Delta t=0.125$, but they are not stable for stepsize $\Delta t=1$. This indicates that the restriction of the stepsize $\Delta t_{0}$ in Theorem 4.3 is not theoretical optimal. From Figure 2, we also can find that the methods behave better stability when the value of $\theta$ increases and the stepsize $\Delta t$ decreases.

\section{Acknowledgments}

The authors thank the anonymous referee and the editors for their detailed comments and helpful suggestions. This work was supported by NSF of China (no.11171352) and Hunan Provincial Innovation Foundation for Postgraduates (no. CX2010B118).

\section{References}

[1] R. Cont and P. Tankov, Financial Modelling with Jump Processes, Financial Mathematics Series, Chapman \& Hall, Boca Raton, Fla, USA, 2004.

[2] N. Bruti-Liberati and E. Platen, "Approximation of jump diffusions in finance and economics," Computational Economics, vol. 29, no. 3-4, pp. 283-312, 2007.

[3] K. Sobczyk, Stochastic Differential Equations. With Applications to Physics and Engineerin, Kluwer Academic, Dordrecht, The Netherlands, 1991.

[4] P. E. Kloeden and E. Platen, Numerical Solution of Stochastic Differential Equations, Springer, Berlin, Germany, 1992.

[5] G. N. Milstein and M. V. Tretyakov, Stochastic Numerics for Mathematical Physics, Springer, Berlin, Germany, 2004.

[6] C. T. H. Baker and E. Buckwar, "Introduction to the numerical analysis of stochastic delay differential equations," Journal of Computational and Applied Mathematics, vol. 125, no. 1-2, pp. 297-307, 2000. 
[7] M. Liu, W. Cao, and Z. Fan, "Convergence and stability of the semi-implicit Euler method for a linear stochastic differential delay equation," Journal of Computational and Applied Mathematics, vol. 170, no. 2, pp. 255-268, 2004.

[8] H. Zhang, S. Gan, and L. Hu, "The split-step backward Euler method for linear stochastic delay differential equations," Journal of Computational and Applied Mathematics, vol. 225, no. 2, pp. 558-568, 2009.

[9] X. Ding, K. Wu, and M. Liu, "Convergence and stability of the semi-implicit Euler method for linear stochastic delay integro-differential equations," International Journal of Computer Mathematics, vol. 83, no. 10, pp. 753-763, 2006.

[10] $\mathrm{P}$. $\mathrm{Hu}$ and C. Huang, "Stability of stochastic $\theta$-methods for stochastic delay integro-differential equations," International Journal of Computer Mathematics, vol. 88, no. 7, pp. 1417-1429, 2011.

[11] W. Wang and Y. Chen, "Mean-square stability of semi-implicit Euler method for nonlinear neutral stochastic delay differential equations," Applied Numerical Mathematics, vol. 61, no. 5, pp. 696-701, 2011.

[12] D. J. Higham and P. E. Kloeden, "Numerical methods for nonlinear stochastic differential equations with jumps," Numerische Mathematik, vol. 101, no. 1, pp. 101-119, 2005.

[13] D. J. Higham and P. E. Kloeden, "Convergence and stability of implicit methods for jump-diffusion systems," International Journal of Numerical Analysis and Modeling, vol. 3, no. 2, pp. 125-140, 2006.

[14] E. Platen and N. Bruti-Liberati, Numerical Solution of Stochastic Differential Equations with Jumps in Finance, vol. 64, Springer, Berlin, Germany, 2010.

[15] X. Wang and S. Gan, "Compensated stochastic theta methods for stochastic differential equations with jumps," Applied Numerical Mathematics, vol. 60, no. 9, pp. 877-887, 2010.

[16] L. Ronghua and C. Zhaoguang, "Convergence of numerical solution to stochastic delay differential equation with Poisson jump and Markovian switching," Applied Mathematics and Computation, vol. 184, no. 2, pp. 451-463, 2007.

[17] L.-s. Wang, C. Mei, and H. Xue, "The semi-implicit Euler method for stochastic differential delay equations with jumps," Applied Mathematics and Computation, vol. 192, no. 2, pp. 567-578, 2007.

[18] N. Jacob, Y. Wang, and C. Yuan, "Numerical solutions of stochastic differential delay equations with jumps," Stochastic Analysis and Applications, vol. 27, no. 4, pp. 825-853, 2009.

[19] D. Liu, "Mean square stability of impulsive stochastic delay differential equations with Markovian switching and Poisson jumps," International Journal of Computational and Mathematical Sciences, vol. 5, no. 1, pp. 58-61, 2011.

[20] J. Tan and H. Wang, "Mean-square stability of the Euler-Maruyama method for stochastic differential delay equations with jumps," International Journal of Computer Mathematics, vol. 88, no. 2, pp. 421-429, 2011.

[21] X. Mao, Stochastic Differential Equations and Applications, Horwood, Chichester, UK, 1997.

[22] S. Mohamad and K. Gopalsamy, "Continuous and discrete Halanay-type inequalities," Bulletin of the Australian Mathematical Society, vol. 61, no. 3, pp. 371-385, 2000.

[23] E. Hairer and G. Wanner, Solving Ordinary Differential Equations. II: Stiff and Differential-Algebraic Problem, Springer, Berlin, Germany, 2nd edition, 1996.

[24] C. T. H. Baker and E. Buckwar, "On Halanay-type analysis of exponential stability for the $\theta$-Maruyama method for stochastic delay differential equations," Stochastics and Dynamics, vol. 5, no. 2, pp. 201-209, 2005. 


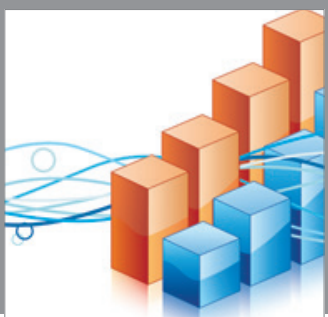

Advances in

Operations Research

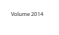

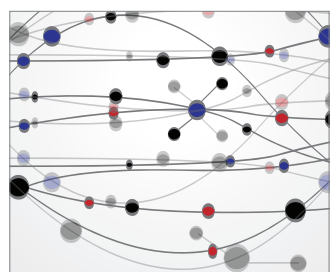

\section{The Scientific} World Journal
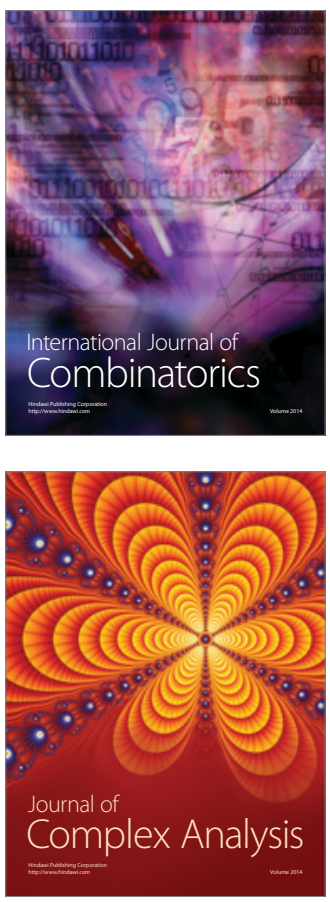

International Journal of

Mathematics and

Mathematical

Sciences
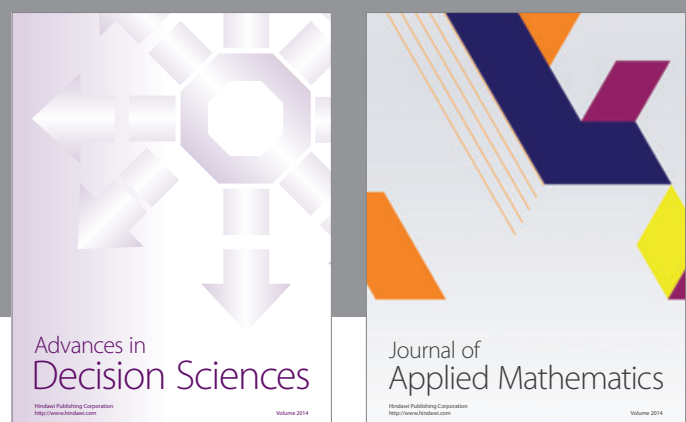

Journal of

Applied Mathematics
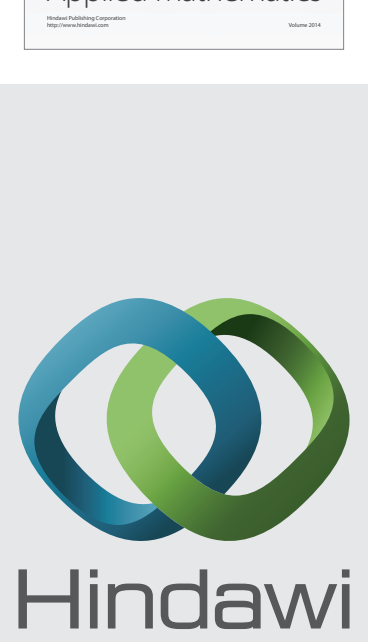

Submit your manuscripts at http://www.hindawi.com
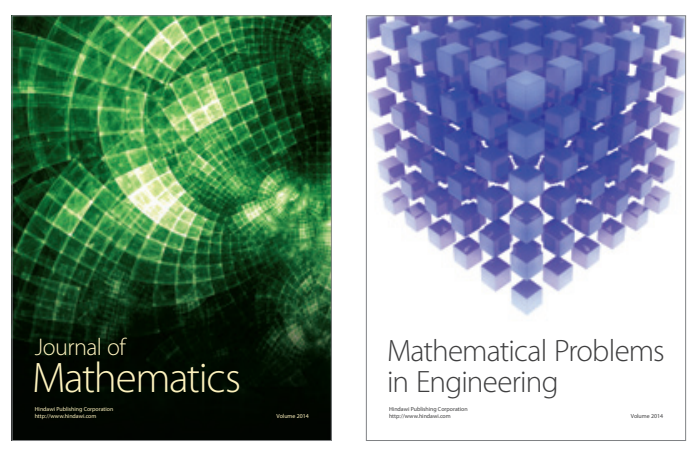

Mathematical Problems in Engineering
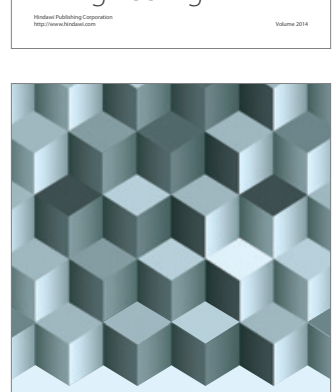

Journal of

Function Spaces
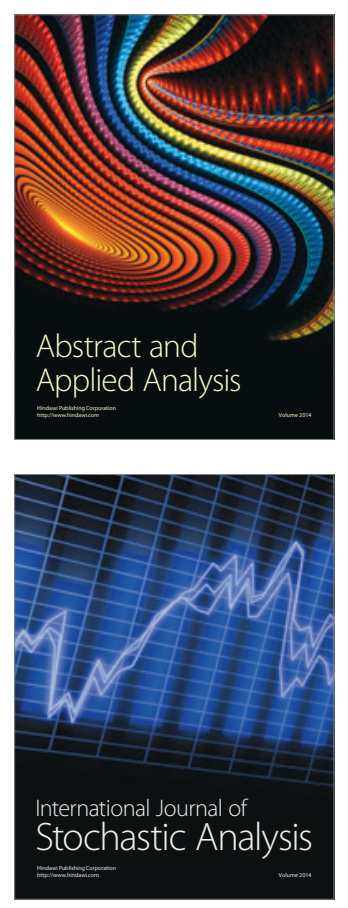

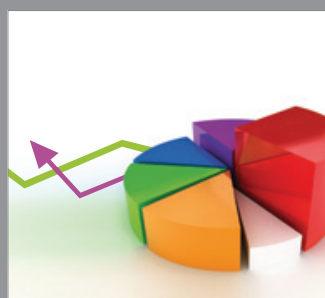

ournal of

Probability and Statistics

Promensencen
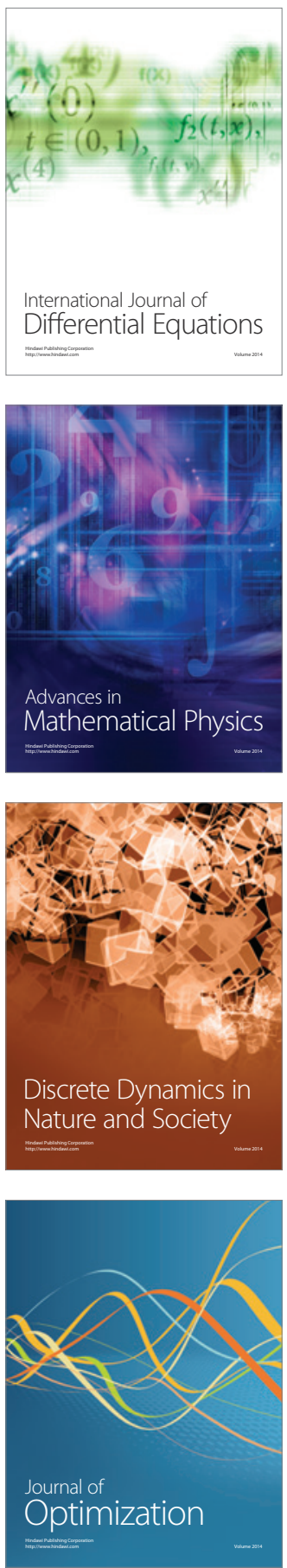